\title{
Assessing The Overall Driving Performance Of Older Drivers
}

\author{
Seddigheh BABAEE, Yongjun SHEN, Elke HERMANS, Tom BRIJS, \\ Geert WETS, Ariane CUENEN
}

Transportation Research Institute, Hasselt University, Wetenschapspark 5, bus 6, 3590 Diepenbeek, Belgium; PH 32(0)11 2691 \{64, 43, 41, 58, 55, 35, 08\}; FAX +32(0)11 2691 99; E-Mail: \{seddigheh.babaee, yongjun.shen, elke.hermans, tom.brijs, geert.wets, Ariane.cuenen\}@ uhasselt.be

\begin{abstract}
This study investigates the relative performance of older drivers at the individual level, based on specific measures of functional and driving abilities. To do so, 55 participants aged 70 years and above completed tests of an assessment battery of psychological and physical aspects as well as knowledge of road signs; moreover, they took a driving simulator test in which specific driving situations that are known to cause difficulties for older drivers were included. To evaluate the overall performance of each driver, all the above information was combined by using the concept of composite indicators, and the technique of data envelopment analysis, which is an optimization model for measuring the relative performance of a set of units, or drivers in this study, was employed. The model output distinguishes the best-performers from those underperforming drivers, and helps in guiding future development of training interventions tailored to each individual by specifically targeting those functions that are (mostly) impaired.
\end{abstract}

KEYWORDS: Older drivers; Overall driving performance; Driving simulator; Composite indicators; Data envelopment analysis.

\section{INTRODUCTION}

Worldwide, the number of persons over 65 years of age is increasing, mainly as a result of improvements in health and longevity. By 2030, one in every 8 of the earth's inhabitants will age above 65 (U.S. National Institute on Aging, 2007). The increase in the population of elderly coincides with an increase in older drivers (Langford and Koppel, 2006). At the same time, the safety and mobility of older drivers are challenged by several age-related changes, including sensory, motor and cognitive abilities and a decline in those aspects affect the ability to drive safely. Moreover, 
when older road users are involved in collisions, the risk of being seriously or fatally injured is higher due to increased frailty with age. Taking the distances travelled into account, the fatality rate for a car driver aged 75 years and over is more than 5 times higher than for the average for all ages, whereas their injury rate is two times higher (European Road Safety Observatory, Older drivers, 2007).

In this study, we aim to quantify the overall driving performance of a sample of older drivers using data from an assessment battery and a fixed-based driving simulator, in order to be able to tailor simulator-based training and psychological and physical training to an individual in the future and specifically target those driving skills and psychological and physical abilities that are impaired. To do so, the concept of composite indicators (CIs), in which various relevant information is combined in one score, is employed, and the technique of data envelopment analysis (DEA) in general, and the multiple layer DEA in particular, is used for the index construction. It is a data-driven approach and to our knowledge, it is the first time that this model is used for the performance evaluation of individual older drivers. The results will enable us to distinguish the best drivers from underperforming ones and to advise drivers with detailed suggestions for their improving performance based on their weakness areas.

We continue in Section 2 with the explanation about the data collection. The methodology is discussed in Section 3. Section 4 deals with the corresponding results in terms of a ranking of the 55 older drivers based on their index scores and an illustration of the most problematic parameter(s) for a particular older driver. Section 5 concludes the paper and offers some final remarks.

\section{DATA COLLECTION}

In this study, we develop an appropriate tool to screen older drivers and assess their relative performance and identify aspects requiring most attention for improvement. We carry out a secondary analysis of data drawn from older persons in a research study conducted by Cuenen et al. (submitted). Data were gathered at 2 places. First, older drivers completed tests of an assessment battery at the Jessa Hospital. Second, simulator driving tests, scheduled on different days but still in the same week, took place at the Transportation Research Institute of Hasselt University.

\section{Participants}

In total, 77 participants aged 70 years or older were recruited largely through the Geriatrics department of the Jessa Hospital, by oral presentation for senior associations and with flyers distributed in senior flats, hospitals and senior 
associations. Drivers were included if they were an active driver with a valid driver's license, with no stroke in the last four months and psychologically healthy - i.e. having a Mini Mental State Examination score above 24 (Iverson et al., 2010) - and the physical ability to complete tests of a clinical assessment battery and simulator driving. Among these participants, 16 suffered from simulator sickness from the start of the experiment, while 6 became sick while carrying out the second ride. As a whole, 22 participants were excluded. Thus, 55 participants (mean age $=76.49$; standard deviation $=5.40$ ) remained in the sample.

\section{Assessment battery}

The validated clinical test battery included tests of psychological and physical abilities as well as knowledge of road signs, and was documented in detail in the study of Cuenen et al. (submitted). For the purpose of this study, we only consider information on the abilities described below.

\section{Psychological ability}

\section{The Mini Mental State Examination (MMSE)}

We used the MMSE to screen for cognitive impairment. The possible score ranges from 0 to 30 . The higher the score, the better the psychological ability.

\section{The Digit Span Forward (DSF)}

We used the DSF which is originally part of the Digit Span Subtest of the Wechsler Adult Intelligence Scale (Wechsler, 1955). In the task, participants repeat a random sequence of numbers in forward direction. It assesses attention and working memory, as well as auditory memory (Clark, et al., 2011). The more numbers a person can repeat, the better the psychological ability. Scores on this tasks are divided in four categories $(0=$ impaired, $1=$ beneath average, $2=$ average, $3=$ above average $)$.

\section{The Useful Field of View (UFOV)}

We used three Useful Field of View sub tests (UFOV 1= Visual processing speed; UFOV 2= divided attention; and UFOV 3 = selective attention) (Edward et al., 2006). Scores are expressed in milliseconds and range from $16.7 \mathrm{~ms}$ to $500 \mathrm{~ms}$ for each subtest. Lower scores correspond with improved visual attention.

\section{Physical ability}

\section{The Snellen chart}

This test is used for measuring visual acuity and is one of the most common

clinical measurements of visual function (Rosser, et al., 2001). The chart consists of 
several lines. Participants have to stand $6 \mathrm{~m}$ from the chart and read the lines. The more lines a person can read, the better the visual acuity (with a maximum score of 1.2).

\section{The Get-Up-and-Go test or Rapid Pace Walk}

This test is also used to measure motor abilities (Carr et al., 2010). Scores on this task are divided into three categories $(0=$ more than 20 seconds, $1=$ between 20 and 11 seconds, $2=$ less than 11 seconds). A faster performance corresponds with a higher motor ability.

\section{The Four-test balance scale}

This test is also used to assess motor abilities; more specifically, lower limb muscle strength and balance, with a maximum score of 1 .

\section{Knowledge of road signs}

The Road Sign Recognition (RSR) test is used to measure the knowledge of participants regarding road signs with a maximum score of 12 (Lundberg et al., 2003).

\section{Simulator driving test}

Driving performance was measured in a fixed-based medium-fidelity driving simulator (STISIM M400; Systems Technology Incorporated) with a force-feedback steering wheel, brake pedal, and accelerator. The visual virtual environment was presented on a large $180^{\circ}$ field of view seamless curved screen, with rear view and side-view mirror images. Three projectors offer a resolution of $1024 \times 768$ pixels on each screen and a $60 \mathrm{~Hz}$ refresh rate. Data were collected at frame rate.

\section{Scenario}

Information on the crash types of older drivers was taken into account and situations were included in the scenarios that are known to be difficult for older drivers. These situations are well documented in the literature. For instance, older drivers are over-represented in crashes occurring while turning off at intersections, where typically the older driver turns against oncoming traffic with right of way on the main road (Hakamies-Blomqvist, 1993; Zhang et al., 1998), gap acceptance while turning left at an intersection (Langford and Koppel, 2006; Yan et al., 2007) and response to signs, signals and road hazards (Bao and Boyle, 2008; Horswill et al., 2010). A detailed description of the driving scenario is mentioned in Cuenen et al. (submitted).

In summary, drivers first acquired with the driving simulator. Thereafter they had two main rides. These rides took place at inner-city $(50 \mathrm{~km} / \mathrm{h})$ sections, outer-city $(70,90 \mathrm{~km} / \mathrm{h})$ sections and highway $(120 \mathrm{~km} / \mathrm{h})$ sections. Several traffic situations were 
included, in a randomized way. For instance, two 4-way intersections with a stop sign appeared within the inner-city segments. Drivers were required to give way and two road works were presented where a car was driving in front of the driver with a speed at least $10 \mathrm{~km} / \mathrm{h}$ beneath the speed limit in two outer-city sections. Due to roadblocks, there was no opportunity to overtake. Cars were calibrated such that crashes could be avoided by braking (when driving at speed limit).

\section{Driving measures}

A total of 3 driving measures or indicators were used in the analysis:

1) Mean-Complete Stop (yes or no) which computed from 200 meters before reaching the stop sign until the location of the stop sign.

2) Average Following Distance in a road with a speed of $50 \mathrm{~km} / \mathrm{h}$ (Ave-FD-50) and $70 \mathrm{~km} / \mathrm{h}$ (Ave-FD-70).

3) Mean driving Speed which is measured across separate road segments (i.e., 4.8 $\mathrm{km}$ ) without any events (Trik et al., 2010) with the posted speed limits of 50, 70,90 and $120 \mathrm{~km} / \mathrm{h}$.

\section{DATA ANALYSIS}

Indicators are helpful tools for monitoring, benchmarking, visualization, etc. (Litman et al., 2005, 2007; OECD, 2001; Salzman, 2003). Recently, various indicators have been combined in so-called composite indicators (CIs) or indexes (e.g., Al-Haji 2007). Simplistically, a composite indicator synthesizes the information included in a selected set of indicators in one score (Nardo et al., 2005). In this study, a composite indicator was created with respect to all aforementioned indicators for older drivers (see Figure 1). It should be mentioned that the driving measures and road sign recognition were considered in a group as driving ability.

First, the performance of older drivers was obtained by means of the corresponding assessment criteria based on literature review. Then, normalization was carried out, because of the different measurement units of the indicators. Among existing methods (Nardo et al., 2005), we used in this study, the Min-Max method which normalizes indicators to have an identical range, [0,1], by subtracting the

minimum value and dividing by the range of the indicators values. Thereafter, for the older-driver performance index construction, Data Envelopment Analysis (DEA) in general, and the Multiple Layer DEA Composite Indicator method in particular is used. Both methodologies are explained in the following section. 


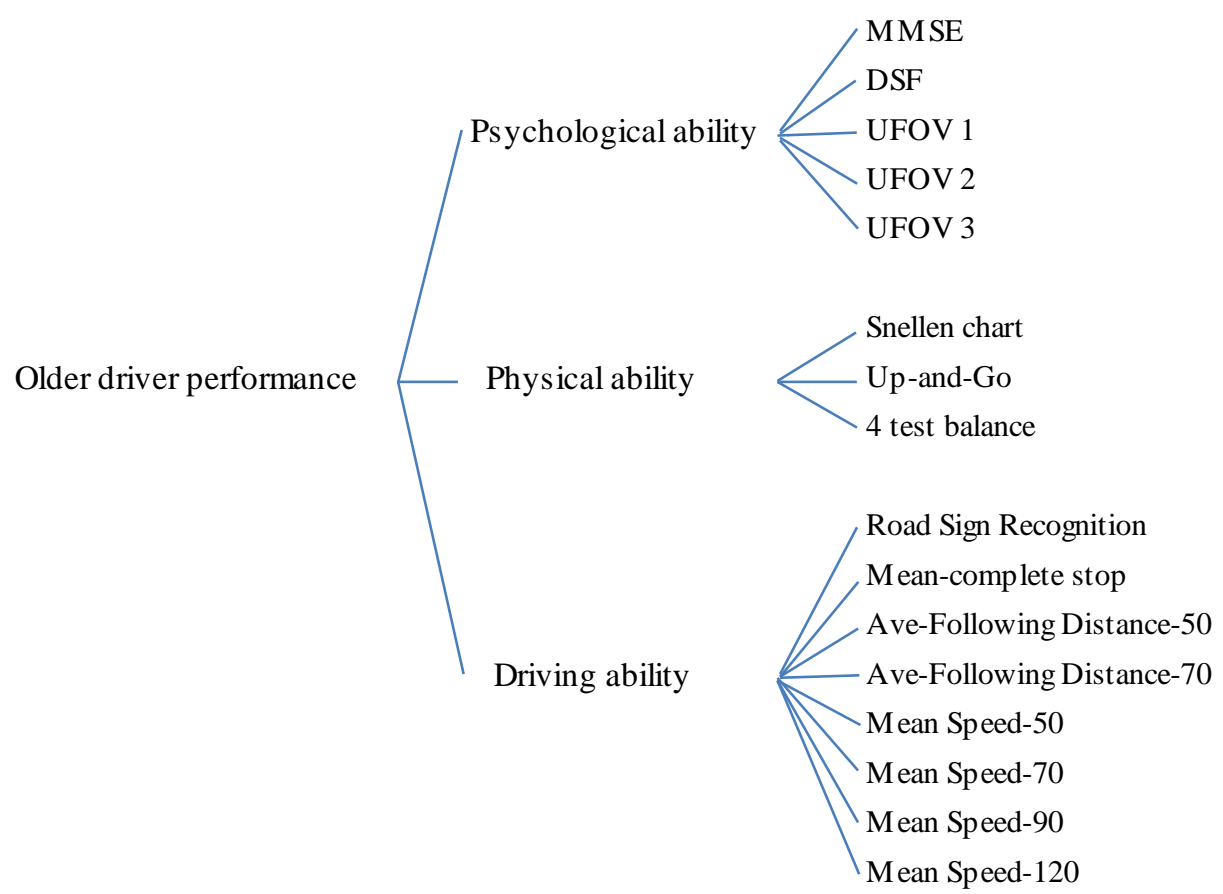

Figure 1. Hierarchical structure of older driver's performance indicators.

\section{Data Envelopment Analysis}

DEA, originally developed by Charnes, Cooper, and Rhodes (1978), is a nonparametric optimization technique that uses linear programming to measure the relative efficiency of a set of decision making units, or drivers in our study. The focus of DEA is on the individual unit, in contrast to the focus on the average of a set of units.

Lately, considerable attention has been paid to the application of DEA in the construction of CIs: The environmental performance index (Färe et al., 2004), the human development index (Despotis, 2005), the sustainable energy index (Zhou et al., 2007) and the road safety performance index (Hermans, 2009) are examples hereof.

In this study, to evaluate the performance of each driver by combining all the hierarchically structured indicators in one index score, a multiple layer DEA based composite indicator model (MLDEA-CI) developed by Shen et al. (2013) is adopted. This model is able to take the layered hierarchy of indicators into account that often exists in reality. The main idea of this model is to first aggregate the values of the indicators within a particular category of a particular layer by the weighted sum approach in which the sum of the internal weights equals one. Then, for the first layer, the weights for all the sub-indexes are determined using the basic DEA approach.

More specifically, suppose that a set of $n$ DMUs is to be evaluated in terms of $s$ 
indicators $(y)$ with a $K$ layered hierarchy, the MLDEA-CI model can be formulated as follows (Shen et al., 2013):

$$
\begin{aligned}
C I_{0}=\max & \sum_{f_{1}=1}^{s} \hat{u}_{f_{1}} y_{f_{1} 0} \\
\text { s.t. } \quad & \sum_{f_{1}=1}^{s} \hat{u}_{f_{1}} y_{f_{1} j} \leq 1, \quad j=1, \cdots, n \\
& \sum_{f_{1} \in A_{f_{k}}^{(k)}} \hat{u}_{f_{1}} / \sum_{f_{1} \in A_{f_{k+1}^{(k+1)}}} \hat{u}_{f_{1}}=w_{f_{k} \in A_{f_{k+1}}^{(k+1)}}^{(k)} \in \Theta, f_{k}=1, \cdots, s^{(k)}, \quad k=1, \cdots, K-1 \\
& \hat{u}_{f_{1}} \geq 0, \quad f_{1}=1, \cdots, s
\end{aligned}
$$

where

$\hat{\mathrm{u}}_{f_{1}}$ is the set of most favorable optimal weights for $\mathrm{DMU}_{0}$, which are obtained by solving the model.

$s^{(k)}$ is the number of categories in the $k^{\text {th }}$ layer $(k=1,2, \ldots, K) . s^{(1)}=s$.

$A_{f_{k}}^{(k)}$ denotes the set of indicators of the $f^{\text {th }}$ category in the $k^{\text {th }}$ layer.

$w_{f_{k}}^{(k)}$ denotes the internal weights associated with the indicators of the $f^{\text {th }}$ category in the $k^{\text {th }}$ layer, which sum up to one within a particular category.

$\Theta$ denotes the restrictions imposed to the corresponding internal weights.

The subscript, o, refers to the driver whose index score is to be obtained by solving the constrained optimization problem, which maximizes the index value of the driver and satisfies the imposed restrictions. The first restriction guarantees an intuitive interpretation of the composite indicator and implies that no driver in the data set can be assigned an index value larger than one under these weights. With respect to the second restriction, the layered hierarchy of the indicators is reflected by specifying the weights in each category of each layer and further restricting their flexibility. In doing so, obtainment of realistic and acceptable weights is guaranteed.

To make sure that all the three aspects of a driver's abilities - psychological, physical and driving abilities - will be represented to some extent in the overall driving performance index score, each of these three factors is considered to have a similar importance in the final index score but still with $30 \%$ variability to allow a high level of flexibility. In addition, by the third restriction, all weights are restricted to be nonnegative. 


\section{RESULTS}

Using the data from the assessment battery and the driving simulator tests - values of 16 driving indicators for each of the 55 drivers - and applying the MLDEA-CI model yields the following results:

\section{Index scores and older drivers ranking}

By applying the model, 16 indicators are now combined in an overall index score for each driver by selecting the best possible indicator weights under the imposed restrictions. As a result, the index score of each older driver is calculated in relation to all the other drivers who took part in the experiment. Index values lie between zero and one with a value equal to one identifying a best performer, whereas a score less than one implies underperforming drivers. Apart from distinguishing the best-performing and underperforming drivers, it is possible to rank them based on their calculated index scores (see Table 1). As can be seen, drivers with ID number 21, 24, 42 and 50 are identified as best performers and the driver with ID number 43 as the worst performer.

Table 1. Older drivers' ranking based on their performance index scores

\begin{tabular}{lcccccccccccc}
\hline Ranking & 1 & 1 & 1 & 1 & 2 & 3 & 4 & $\ldots$ & 49 & 50 & 51 & 52 \\
Driver's ID & 21 & 24 & 42 & 50 & 9 & 54 & 8 & $\ldots$ & 10 & 33 & 52 & 43 \\
Index score & 1 & 1 & 1 & 1 & 0.995 & 0.993 & 0.980 & $\ldots$ & 0.664 & 0.658 & 0.644 & 0.640 \\
\hline
\end{tabular}

\section{Weight allocation and required improvement priorities}

In addition to the ranking of the drivers, more detailed insight can be gained from the assigned weights which can be interpreted as indications of the importance shares of the corresponding indicator. Along with tracking the optimal index score for each individual, the model guarantees acceptable weights through the imposed restrictions. Figure 2 shows the assigned weights (last column) and shares (percentages in the middle) for the case of the worst driver in the data set.

As can be seen, the performance with respect to all three performance categories is taken into account in the overall score with the share of psychological ability equal to $36.36 \%$, that of physical ability $27.61 \%$ and that of driving ability $36.03 \%$. 


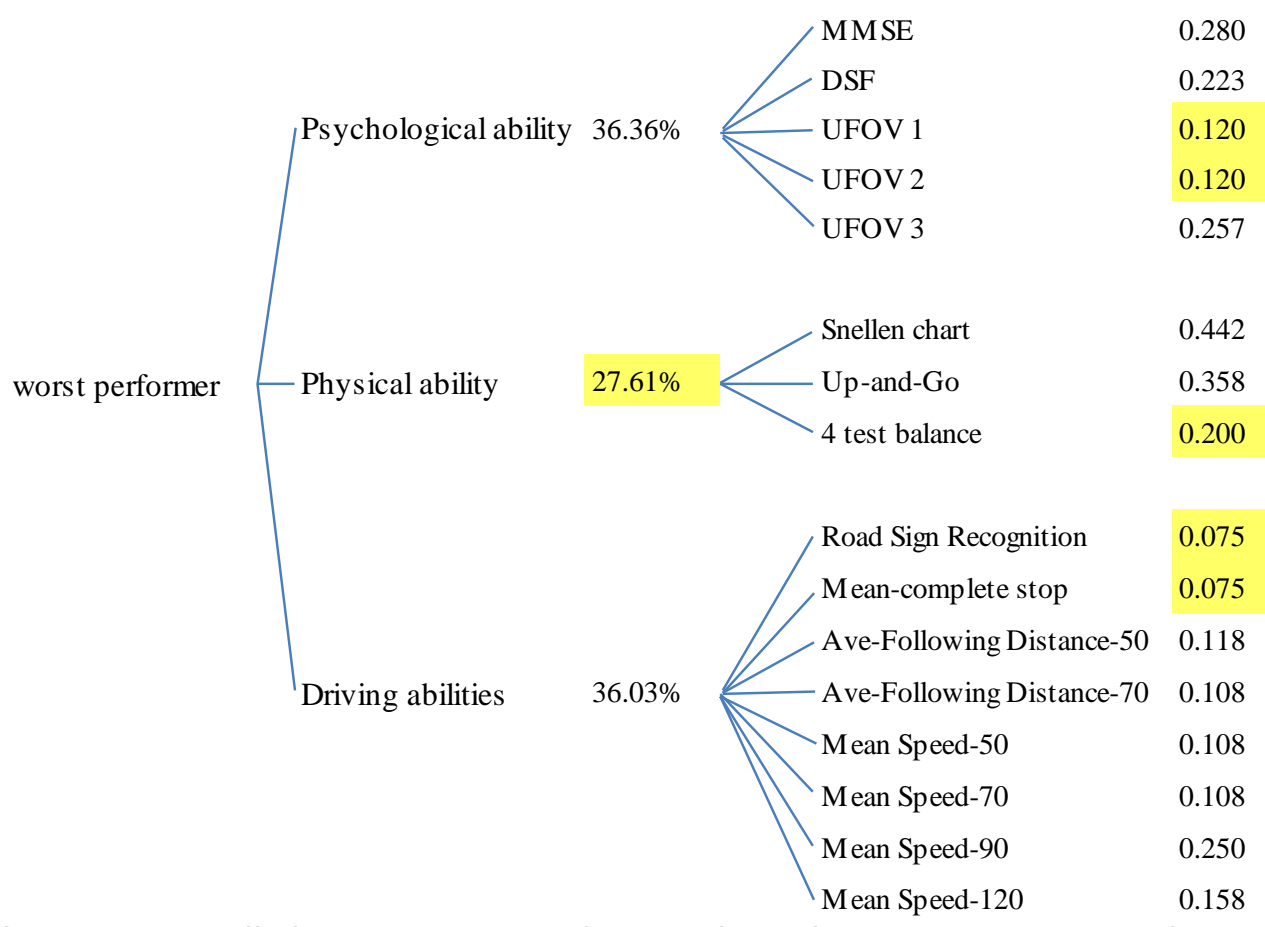

Figure 2. Assigned weights and shares from the model for the case of the worst performer.

More importantly, based on the principle of the maximization MLDEA-CI model, an indicator is assigned a high weight if the driver performs relatively well on that aspect. On the contrary, low weights provide us with valuable information about the aspects requiring most attention for improvement. Therefore, areas of underperformance can be detected, and required improvement priorities can be formulated. Taking the indicators of psychological, physical and driving abilities related to the worst performer as an example, it can be seen that this person is doing relatively well with respect to the psychological aspect (with the highest share of $36.36 \%$ ) whereas more attention should be paid to the physical ability (with the lowest share of $27.61 \%$ ), by focusing on motor ability, more specifically lower limb muscle strength and balance, as the lowest weight within this category is assigned to the 4 test balance (0.200). Improvement priorities can also be given within the psychological abilities to UFOV $1 \& 2$ aspects and within driving abilities to Road Sign Recognition and Mean-complete stop. 


\section{CONCLUSION}

In this study, an assessment battery was used to evaluate older drivers' psychological and physical abilities as well as knowledge of road signs. In addition, by using a driving simulator, the driving performance of participants was measured in situations that are especially difficult for older drivers. Then, all this information was aggregated to construct an overall performance index score in order to quantify the relative performance of individual older drivers. In doing so, a multiple layer DEA based composite indicator model was applied. Based on this model,

- the most optimal performance index score between zero and one for each of the 55 older drivers was determined by combining all the 16 hierarchical indicators, based on which the best performing drivers - having an index score of one - were deduced. At the same time, underperforming drivers were revealed.

- Apart from identifying the best performing and underperforming drivers, their relative performance with respect to psychological abilities, physical abilities and driving performance was compared.

- Based on the principle of the MLDEA-CI model, areas of underperformance could be detected for each individual, and required future improvement priorities formulated.

To conclude, this study suggests that the MLDEA-CI methodology is appropriate for older driver's evaluation and for the identification of the most problematic aspects of their performance. Future research on the composite driving performance indicator in case of older drivers can be done concerning methodological challenges ,e.g., including more higher cognitive functions such as hazard perception and change blindness and sensitivity analysis of the index score.

\section{REFERENCES}

Al-Haji, G. (2007). "Road Safety Development Index (RSDI): Theory, Philosophy and Practice." Department of Science and Technology. PhD Dissertation, Campus Norrköping, Linköping University, Norrköping.

Bao, S., and Boyle, L. (2008). "Driver Performance at Two-Way Stop-Controlled Intersections on Divided Highways." Transportation Research Record, 2069, 26-32.

Carr, D. B., Schwartzberg, J. G., Manning, L., and Sempek, J. (2010). "Physician's guide to assessing and counseling older drivers." 2nd edition. Washington D.C.: National Highway Traffic Safety Administration.

Cuenen, A., Jongen, E., Brijs, T., Brijs, K., Lutin, M., Van Vlierden, K., van Breukelen, 
G. and Wets, G. (2012). "Beyond summarized measures: predictability of specific measures of simulated driving by specific physical and psychological measures in older drivers."

Charnes A., Cooper W. W. and Rhodes E. (1978). "Measuring the efficiency of decision making units." European Journal of Operational Research, 2, 429444.

Despotis, D.K. (2005). "A reassessment of the human development index via data envelopment analysis." Journal of the Operational Research Society, 56, No. 8, 969-980.

Edwards, J. D., Vance, D. E., Wadley, V. G., Cissell, G. M., Roenker, D. L. and Ball, K. (2005). "Reliability and validity of Useful Field of View Test scores as administered by personal computer." Journal of Clinical and Experimental Neuropsychology, 27, 529-543.

European Road Safety Observatory, Older Driver. http://ec.europa.eu/transport/road_safety/specialist/knowledge/old/index.htm

Färe, R., Grosskopf, S. and F. Hernandez-Sancho (2004). "Environmental Performance: An Index Number Approach." Resource and Energy Economics. 26 (4), 343-352.

Hakamies-Blomqvist, L. E. (1993). "Fatal accidents of older drivers." Accident Analysis and Prevention, 25, 19-27.

Hermans, E. (2009). "A methodology for developing a composite road safety performance index for cross-country comparison." $\mathrm{PhD}$ thesis, Hasselt university, Hasselt, Belgium.

Horswill, M.S., Kemala, C. N., Wetton, M., Scialfa, C.T. and Pachana, N.A. (2010). "Improving older drivers' hazard perception ability." Psychology and Aging, 25, 464-469.

Langford, J. and Koppel, S. (2006). "Epidemiology of older driver crashes Identifying older driver risk factors and exposure patterns." Transportation Research Part F, 9, 309-321.

Litman, T. (2005). "Well measured: Developing indicators for comprehensive and sustainable transport planning." Victoria Transport Policy Institute.

Litman, T. (2007). "Developing indicators for comprehensive and sustainable transport planning." Presented at the 86th annual meeting of the Transportation Research Board, Washington D.C.

Lundberg, C., Caneman, G., Samuelsson, S., Hakamies-Blomqvist, L. and Almkvist, O. (2003). "The assessment of fitness to drive after a stroke: The Nordic Stroke 
Driver Screening Assessment.” Scandinavian Journal of Psychology, 44, 23-30.

Nardo, M., Saisana, M., Saltelli, A., Tarantola, S., Hoffman, A. and Giovannini, E. ( 2005b). "Handbook on constructing composite indicators: Methodology and user guide." Organisation for Co-operation and Development.

Organisation for Economic Co-operation and Development (2001). "Performance indicators for the road sector: Summary of the field tests." Organisation for Economic Co-operation and Development.

Rosser, D. A., Laidlaw, D. A. H. and Murdoch, I. E. (2001). "The development of a "reduced $\log$ MAR" visual acuity chart for use in routine clinical practice." British Journal of Ophthalmology, 85(4), 432-436.

Salzman, J. (2003). "Methodological choices encountered in the construction of composite indices of economic and social well-being." Center for the Study of Living Standards.

Shen, Y., Hermans, E., Brijs, T. and Wets, G. (2013). “ Data envelopment analysis for composite indicators: A multiple layer model." Social Indicators Research, 114, No. 2, 739-756.

Trik, L. M., Toxopeus, R. and Wilson, D. (2010). "The effect of visibility, traffic density and navigational challenge on speed compensation and driving performance in older adults." Accident Analysis and Prevention. 42, 16611676.

U.S. National Institute on Aging, National Institute of Health (March 2007). "Why population aging matters, a global perspective". publication No. 07-6134.

Wechsler, D. (1955). "Manual for the Wechsler Adult Intelligence Scale." Oxford, England: Psychological Corporation

Yan, X., Radwan, E. and Guo, D. (2007). "Effects of major-road vehicle speed and driver age and gender on left-turn gap acceptance." Accident Analysis and Prevention, 39, 843-852.

Zhang, J., Fraser, S., Clarke, K. and Mao, Y. (1998). "Age-specific patterns of factors related to fatal motor vehicle traffic crashes: focus on young and elderly drivers." Public Health, 112, 289-295.

Zhou, P., Ang, B.W. and Poh, K.L. (2007) "A mathematical programming approach to constructing composite indicators." Journal of the Ecological Economics, 62, 291-297. 